Service social

\title{
Soutien à la transition vers la vie adulte : étude exploratoire auprès de jeunes présentant une déficience physique
}

\author{
Normand Boucher, Marie-Ève Schmouth, Andrée-Anne Lizotte, Sylvie \\ Tétreault, J. Côté et M. Couture
}

Volume 62, numéro 2, 2016

URI : https://id.erudit.org/iderudit/1038580ar

DOI : https://doi.org/10.7202/1038580ar

Aller au sommaire du numéro

\section{Éditeur(s)}

École de travail social et de criminologie de l’Université Laval

ISSN

1708-1734 (numérique)

Découvrir la revue

Citer cet article

Boucher, N., Schmouth, M.-È., Lizotte, A.-A., Tétreault, S., Côté, J. \& Couture, M. (2016). Soutien à la transition vers la vie adulte : étude exploratoire auprès de jeunes présentant une déficience physique. Service social, 62(2), 114-130. https://doi.org/10.7202/1038580ar

\section{Résumé de l'article}

Les jeunes ayant des incapacités sont souvent limités sur le plan du développement d'habiletés essentielles au passage à la vie adulte. Cet article présente les retombées d'une intervention de groupe (durée de 12 mois) auprès de 10 jeunes âgés de 16 à 21 ans et visant à favoriser le développement de telles habiletés. Les analyses statistiques portant sur les données recueillies auprès des jeunes et de leurs parents ont montré des différences positives significatives ( $p$ \&lt; 0,05$)$ entre le début et la fin de l'intervention $(\mathrm{T} 0, \mathrm{~T} 2)$ sur la capacité des jeunes à réaliser certaines habitudes de vie (déplacements dans la communauté, responsabilités, aspects associés à leur alimentation et à leurs soins personnels). L'intervention aurait eu un effet similaire sur leur sentiment d'efficacité personnelle, mais non au plan de l'estime d'eux-mêmes. Ces résultats sont probablement en partie l'effet de l'approche utilisée (centrée sur les jeunes et axée sur les apprentissages in vivo), qui pourrait être à préconiser avec cette clientèle. 


\section{Soutien à la transition vers la vie adulte : étude exploratoire auprès de jeunes présentant une déficience physique}

BOUCHER, Normand, Ph. D sociologie Professeur associé de l'École de service social à l’Université Laval Chercheur régulier au Centre interdisciplinaire de recherche en réadaptation et intégration sociale et chercheur d'établissement du CIUSSS de la Capitale-Nationale

SCHMOUTH, Marie-Ève, M.A., baccalauréat et maîtrise en sociologie Professionnelle de recherche au Centre interdisciplinaire de recherche en réadaptation et intégration sociale du CIUSSS de la Capitale-Nationale

LIZOTTE, Andrée-Anne

Éducatrice spécialisée

Programme de soutien à l'intégration sociale au CIUSSS de la Capitale-Nationale

TÉTREAULT, Sylvie, Ph. D service social Professeure à la Haute École de santé et de travail social EESP Professeure associée au Département de réadaptation de l'Université Laval Chercheuse au Centre interdisciplinaire de recherche en réadaptation et intégration sociale du CIUSSS de la Capitale-Nationale

\section{CôTÉ, J.}

Éducatrice spécialisée Professeure au Cégep de Ste-Foy en Techniques d'éducation spécialisée

COUTURE, M., M. Sc., ergothérapeute Conseillère à la recherche à la Direction de la qualité, de la performance et des mandats universitaires du CIUSSS de la Capitale-Nationale

\section{RÉSUMÉ}

Les jeunes ayant des incapacités sont souvent limités sur le plan du développement d'habiletés essentielles au passage à la vie adulte. Cet article présente les retombées d'une intervention de groupe (durée de 12 mois) auprès de 10 jeunes âgés de 16 à 21 ans et visant à favoriser le développement de telles habiletés. Les analyses statistiques portant sur les données recueillies auprès des jeunes et de leurs parents ont montré des différences positives significatives $(p<$ $0,05)$ entre le début et la fin de l'intervention (T0, T2) sur la capacité des jeunes à réaliser certaines habitudes de vie (déplacements dans la communauté, responsabilités, aspects associés à leur alimentation et à leurs soins personnels). L'intervention aurait eu un effet similaire sur leur sentiment d'efficacité personnelle, mais non au plan de l'estime d'eux-mêmes. 
Ces résultats sont probablement en partie l'effet de l'approche utilisée (centrée sur les jeunes et axée sur les apprentissages in vivo), qui pourrait être à préconiser avec cette clientèle.

Mots clés : jeunes ayant des incapacités, transition, vie adulte, intervention de groupe, habitudes de vie

\begin{abstract}
Young adults with disabilities have fewer opportunities than others of the same age group to develop useful social skills needed during the transition into adulthood. This paper introduces the outcomes of a group intervention (12 months) focusing on the development of such social skills and involving 10 young adults aged from 16 to 21 . Statistical analysis of data gathered from participants and their parents shows a significant positive difference $(p<0.05)$ between the beginning and the completion of the intervention (T0-T2) regarding their possibility to achieve some life habits (mobility, responsibilities, nutrition, personal care). The intervention also improved $(p<0.05)$ their self-efficacy, but not their self-esteem. These results are probably partially explained by the chosen mean of intervention (youth centered and focusing on in vivo learnings) that could be preferred with this profile of young adults with disabilities.
\end{abstract}

Keywords: Young adults with disabilities, transition, adulthood, group interventions, life habits 


\section{INTRODUCTION}

En raison des avancées médicales et sociales, la majorité des individus ayant des incapacités atteignent l'adolescence et l'âge adulte (Gibson et al., 2014; Sattoe et al., 2014). Selon des données provenant de Ressources humaines et Développement des compétences Canada, il y aurait 96060 personnes âgées entre 15 et 19 ans qui vivraient avec des incapacités au Canada (Stewart et al., 2013). Cette situation pose la question sociale de ce qu'est et de ce que sera pour ces personnes le processus de transition vers la vie adulte.

De manière générale, cheminer vers la vie adulte signifie acquérir de manière progressive des habiletés, des privilèges, des responsabilités et des caractéristiques qui correspondent aux normes socioculturelles associées au fait d'être un adulte (Gibson et al., 2014). Terminer ses études, trouver un travail, acquérir l'indépendance et développer la capacité à décider pour soimême sont des aspects qui jalonnent le parcours vers la vie adulte (Gibson et al., 2014). Lorsqu'ils discutent de divers aspects associés à leur développement et à leur cheminement, il ressort que les adolescents ayant des incapacités épousent, tout comme les autres, les normes socioculturelles associées à la vie adulte. En effet, ils expriment des aspirations, des intérêts et des désirs typiques pour leur âge, par exemple le succès scolaire, se trouver un emploi, sortir avec des amis, apprendre à se connaître et à se sentir bien avec eux-mêmes ou avec des pairs ou encore avoir des relations sexuelles (East et Orchard, 2014; Gibson et al., 2014). Certains développent aussi une vision d'une transition réussie vers la vie adulte similaire à celle des adolescents sans incapacité, prenant la forme d'un parcours allant des études secondaires au marché du travail. L'indépendance est aussi perçue comme une possibilité atteignable, minimisant la différence que créent les incapacités, mais nécessitant d'abandonner les soins prodigués par l'entourage (Gibson et al., 2014).

D'ailleurs, l'acquisition de ces aspects associés au fait d'être un adulte produit des résultats positifs chez ceux-ci. À ce titre, des chercheurs ont démontré qu'obtenir et maintenir un emploi significatif leur procure des bénéfices, tels que la sécurité d'emploi et l'autosuffisance, mais aussi un statut social, une manière de trouver sa place en société, de construire des relations et une identité sociale et personnelle (Doren et al., 2013; Gibson et al., 2014). De plus, ils ont démontré qu'obtenir et maintenir un emploi significatif, est associé à des niveaux plus élevés d'estime de soi, de sentiment d'efficacité personnelle, de bien-être et de qualité de vie chez ces jeunes (Doren et al., 2013; Gibson et al., 2014). De manière générale, les personnes qui font preuve d'une plus grande autonomie sur le plan de la participation sociale montrent un sentiment plus élevé d'efficacité personnelle (Sattoe et al., 2014).

Dans le contexte actuel, le processus de progression vers la vie adulte pour les adolescents ayant des incapacités est souvent limité, dû à la présence de facteurs multiples, tels que leurs incapacités spécifiques et une offre de services souvent inadéquate (Gibson et al., 2014). En raison de leurs incapacités et de leurs déficiences, de nombreux adolescents demeurent dépendants des parents pour la réalisation de plusieurs habitudes de vie (Stevenson et al., 1997). Mais, un des facteurs environnementaux les plus significatifs est sans aucun doute le peu d'attentes entretenues face aux accomplissements possibles de ces jeunes dans plusieurs aspects de la vie. De cette situation résulte souvent un manque d'occasions de s'impliquer ou d'exercer un contrôle dans diverses activités de la vie quotidienne, jugés insignifiantes ou 
irréalistes pour des jeunes avec des incapacités (East et Orchard, 2014). Le peu d'attentes se manifeste par la présence d'attitudes de surprotection ou de vigilance constante de la part des parents (Betz et Redcay, 2002; Blum, 1995; Reiter et Goldman, 1999; Skär, 2003), et même d'opposition face aux initiatives d'autonomie des individus (Betz et Redcay, 2002; Blum, 1995), ainsi que par la présence d'attitudes discriminatoires (Stewart et al., 2013). Les adolescents ayant des incapacités ont souvent moins de possibilités comparativement à leurs pairs sans incapacité au plan du développement des habiletés cognitives et comportementales, fondamentales à l'exercice de la vie adulte et à la réalisation d'activités de la vie quotidienne (transport, poursuite des études, travail, loisirs, relations amoureuses, vie indépendante) (Betz et Redcay, 2002; Blum, 1995; Doren et al., 2013; East et Orchard, 2014; Gibson et al., 2014; Keenan et al., 2014; Sattoe et al., 2014).

II existe une multitude de stratégies d'intervention visant à les soutenir dans le développement de compétences nécessaires à la transition vers la vie adulte et à la participation sociale (Keenan et al., 2014; Sattoe et al., 2014). Parmi celles-ci, l'Institut de réadaptation en déficience physique de Québec, maintenant fusionné au Centre intégré universitaire de santé et services sociaux de la Capitale-Nationale (CIUSSS de la Capitale-Nationale - IRDPQ), offre aux adolescents et jeunes adultes (16-21 ans) des interventions individuelles et de groupe liées au "Soutien à la transition vers la vie adulte ». Ces interventions ont pour but de les accompagner vers les services offerts aux adultes et de faciliter l'intégration à la communauté. Des interventions destinées aux familles sont aussi dispensées par les intervenants afin de favoriser le soutien des personnes et le respect du cheminement individuel. De plus, les intervenants ont aussi développé des collaborations avec les partenaires du réseau et de la communauté. Sur la base d'observations cliniques et des propos des usagers et de leur famille, recueillis sur une période de deux ans, il semblerait que la participation aux différentes activités proposées ait un effet positif sur le développement de l'autonomie, sur la réalisation de certaines habitudes de vie (p. ex. : utiliser le transport en commun) ainsi que sur la socialisation (p. ex. : échanges plus fréquents entre les participants, rencontres en dehors des activités proposées). Quant à elles, les interventions auprès des familles permettraient aux proches d'avoir une plus grande compréhension du développement et des capacités de leur enfant. Ces bénéfices observés ne sont toutefois pas fondés sur un mode d'évaluation rigoureux.

Le but de la présente étude était de documenter et d'évaluer de manière plus systématique les retombées des interventions de groupe offertes dans le cadre des services de "Soutien à la transition vers la vie adulte » du point de vue des participants et de leur famille. Les informations obtenues devaient constituer le point de départ d'une réflexion de la part des intervenants et des gestionnaires à l'endroit des modalités d'intervention. L'étude émane d'ailleurs de ces acteurs et a été réalisée grâce au Programme de soutien aux cliniciens pour la recherche. 


\section{Méthode}

Devis

Le devis de recherche utilisé était mixte et comprenait des questionnaires et des observations (journal de bord et grilles objectives). Seules les données recueillies à l'aide des questionnaires sont présentées par les auteurs dans cet article.

\section{Participants et procédure}

La population visée était constituée d'adolescents et de jeunes adultes âgés de 16 à 21 ans, ayant reçu un diagnostic de déficience physique, sans déficience intellectuelle modérée ou sévère ${ }^{1}$, et recevant des interventions de "Soutien à la transition vers la vie adulte » destinées à leur groupe d'âge au CIUSSS de la Capitale-Nationale - IRDPQ (n=13), ainsi que leurs parents. II n'était pas nécessaire que parents et enfants d'une même famille participent. Une des intervenantes du programme leur a présenté l'étude. La participation était volontaire et le refus n'affectait en rien les services offerts ou prodigués. Le comité d'éthique de la recherche du CIUSSS de la Capitale-Nationale- IRDPQ a approuvé le projet (Certificat $n^{\circ} 2011-235$ ).

\section{Description des interventions de groupe}

Les objectifs poursuivis par les interventions étaient : 1) de parfaire les éléments travaillés en interventions individuelles dans un contexte de groupe (communication, expression des besoins, planification d'activités, habiletés sociales); 2) de préparer les jeunes à intégrer des groupes déjà existants dans la communauté et 3) de développer des habiletés cognitives et comportementales fondamentales à l'exercice de la vie adulte (p. ex. : habiletés relationnelles et sociales, autonomie, sens des responsabilités). Dans cette visée, les responsables ont planifié des ateliers de même que des activités dans la communauté. Sur le plan des hypothèses, l'équipe postulait que les participants aux interventions de groupe seraient davantage à même de réaliser leurs habitudes de vie à la fin (T2) comparativement au début de l'étude (T0). Elle postulait également que leur sentiment d'efficacité personnelle et leur estime de soi se seraient améliorés à la fin (T2) comparativement à l'entrée de l'étude (T0). Aucune intervention n'a cependant ciblé directement ces aspects.

La participation aux différentes activités était volontaire, sans minimum de participation attendue. Les activités se déroulaient au rythme d'une journée aux deux semaines, habituellement le samedi ou le soir en raison de la fréquentation scolaire pour une période de 12 mois et cela seulement pour les fins de l'étude. Le critère de fin était l'âge, soit 21 ans. Au total, 25 activités bimensuelles d'une durée d'environ trois heures chacune ont été réalisées. Six d'entre elles ont eu lieu dans l'établissement (p. ex.: ateliers de cuisine, activités de connaissances de soi) et 19 se sont déroulées dans la communauté (p. ex. : assister à une partie de hockey, magasinage, cabane à sucre). L'intervenante responsable du groupe lançait une invitation à participer par courriel. L'idée de l'activité venait souvent des participants, à

\footnotetext{
${ }^{1}$ Cette donnée a été confirmée sur la base d'une évaluation en neuropsychologie effectuée avant l'entrée dans le programme.
} 
l'occasion de l'éducatrice. Les participants avaient la responsabilité de répondre à l'invitation afin de confirmer ou non leur présence en respectant une date butoir. Ils avaient aussi pour responsabilité de planifier leurs déplacements ainsi que leur budget pour réaliser l'activité. Certains étaient soutenus au départ en suivi individuel pour faire les apprentissages, notamment relativement aux trajets d'autobus et à la réservation du transport adapté, en vue de participer aux activités du groupe.

Les interventions de groupe et celles réalisées auprès des parents ont été offertes par une équipe composée d'éducateurs spécialisés, d'un travailleur social et de bénévoles âgés d'une vingtaine d'années (pour les adolescents et jeunes adultes seulement). Dans le cadre des interventions de groupe, les intervenants et les bénévoles agissaient à titre de rôle modèle, cherchant à mettre l'accent sur les comportements à favoriser. Les intervenants mettaient de l'avant des principes tels que l'ouverture aux intérêts des participants, l'ajustement aux besoins individuels et à la dynamique du groupe, de même que la responsabilisation et l'implication des individus, afin qu'ils puissent communiquer, planifier et gérer les démarches à faire, prendre des décisions, faire des choix et en assumer les conséquences. Les activités ont été déterminées à mesure que l'intervention se déroulait.

\section{Variables mesurées et outils utilisés}

Lors d'une séance d'évaluation, trois questionnaires ont été administrés aux jeunes, mesurant les variables suivantes: le niveau de réalisation des habitudes de vie, le sentiment d'efficacité personnelle et l'estime de soi. Les chercheurs ont mesuré ces variables à l'entrée dans l'étude (T0), six mois après le début des interventions (T1) et à la fin des interventions (T2). Le questionnaire mesurant la capacité à réaliser certaines habitudes de vie a également servi à évaluer la perception des parents lors des trois temps de mesure. Les données devaient servir à comparer les points de vue de ces deux types d'acteurs et à appuyer possiblement l'hypothèse voulant que l'intervention ait un effet positif sur cette variable. L'équipe de recherche a obtenu les données sociodémographiques (âge, sexe, plus haut niveau de scolarité atteint, occupation principale, source de revenu principale) des participants et des parents au début de l'étude (T0). Un intervenant non impliqué dans les activités du groupe a fait compléter tous les questionnaires à domicile entre octobre 2011 et juin 2012. Les évaluations étaient d'une durée d'environ 90 minutes au T0, puis d'environ 60 minutes pour les temps de mesure subséquents.

Les membres de l'équipe de recherche ont identifié certaines habitudes de vie de la Mesure des habitudes de vie (MHAVIE 4.0) (Fougeyrollas et al., 2011; Fougeyrollas et al., 2012) comme des aspects pertinents à évaluer dans le cadre des interventions : la communication (9 items), les déplacements (6 items), la nutrition (4 items), les soins personnels et de santé (6 items), l'habitation ( 1 item), les responsabilités (4 items), les relations interpersonnelles (3 items), le travail (3 items) et les loisirs (8 items). Plusieurs de ces activités correspondaient à des aspects ciblés par les interventions de groupe. La mesure repose sur le niveau de difficulté et le type d'aide requis, aide technique, aménagement et aide humaine dans la réalisation des habitudes de vie. Ces variables permettent de déterminer un score entre 0 et 18 pour chaque variable $(0=$ une absence de difficulté et d'aide requise à la réalisation des habitudes; $18=$ une grande difficulté et une aide importante à réaliser les habitudes de vie). Une activité non réalisée avait 
un score de 20. Le score a été reporté sur 10 et inversé pour illustrer la réalisation telle que mesurée dans les versions précédentes de la MHAVIE (score obtenu moins 20 et divisé par deux) $(0=$ la présence de perturbations sévères et $10=$ une réalisation optimale des habitudes de vie). Selon une expérimentation de la version 4.0 de la MHAVIE auprès de 400 participants, cette échelle possède de bonnes qualités psychométriques (Boucher et al., 2013).

Le sentiment d'efficacité personnelle fait référence à la croyance qu'entretient une personne envers sa capacité à produire un ou des résultats désirés (Bandura, 2004 dans Keenan et al., 2014). Les chercheurs de l'équipe ont mesuré le sentiment d'efficacité personnelle à l'aide de la version francophone de la Self Efficacy Scale (SES) (Sherer et al., 1982). Le SES est un questionnaire comportant 23 items, divisés en deux sous-échelles. L'une mesure les perceptions d'efficacité personnelle en général (General Self-efficacy) et l'autre en contexte social (Social Self-efficacy). Une échelle de Likert en cinq (5) points permet d'y répondre (absolument pas d'accord à tout à fait d'accord). Des études de validation ont permis de montrer que les deux sous-échelles possèdent une bonne cohérence interne (Sherer et Adams, 1983; Sherer et al., 1982). La validité de critère du SES tend également à être confirmée selon Genest (Genest, s.d.). Dans la présente étude, l'équipe a utilisé une traduction et adaptation du SES par O. Chambon. Cette version comprenait 21 items, dont quatorze mesurant le sentiment d'efficacité général et sept le sentiment d'efficacité social $(0=$ sentiment d'efficacité nul; $5=$ sentiment d'efficacité maximal). Le score a été reporté sur 5 pour les deux sous-échelles (score obtenu divisé par le nombre d'items).

L'estime de soi se rapporte à la perception que l'individu a de lui-même et il a été mesurée à l'aide de la version francophone du Rosenberg Self-Esteem Scale (Dorval, 1996; Rosenberg, 1965). Cette échelle comprend dix questions et une échelle de réponse en quatre points (de " complètement en accord » à " complètement en désaccord ") (score total maximal de 40 points, ramené sur quatre en divisant le score total par le nombre d'items). Les chercheurs considèrent cet outil comme un standard de mesure globale d'estime de soi largement utilisé (Blascovich et Tomaka, 1991), et des études ont démontré certaines de ses qualités psychométriques (Fleming et Courtney, 1984; Magill-Evans, 1993).

\section{Analyses statistiques}

Les chercheurs ont réalisé des analyses non paramétriques pairées pour des mesures répétées dans un même échantillon (Wilcoxon) afin de déterminer l'existence de différences significatives entre le début et la fin de l'intervention (T0, T2) pour les variables mesurées. Ils n'ont pas analysé les données au T1 puisque le but de l'étude était de déterminer les retombées de l'intervention dans sa totalité et non à mi-parcours. Les chercheurs ont considéré, s'appuyant sur les pratiques courantes en analyse statistique, les valeurs $p$ inférieures à 0,05 comme significatives (test exact unilatéral retenu selon les hypothèses de travail). Ils ont réalisé toutes les analyses avec le logiciel SPSS 13. 


\section{Résultats}

\section{Description de l'échantillon}

Au total, 22 personnes ont accepté de participer à l'étude (trois refus chez les jeunes et un parmi les parents). Les jeunes étaient au nombre de dix et étaient âgées de 18 à 21 ans $(X=$ 19,6 ans). La plupart étaient des hommes $(n=6)$ et avaient fréquenté l'école secondaire $(n=7)$. La plupart étaient toujours aux études à temps complet ou à temps partiel $(n=8)$ (tableau 1). Certains avaient des activités secondaires de stage, de travail adapté, de bénévolat ou étaient en recherche d'emploi (résultats non montrés). La majorité des parents, soit 11 sur 12, étaient des femmes et âgées en moyenne de 50,8 ans. La plupart avaient atteint un niveau de scolarité secondaire général, professionnel ou collégial $(n=9)$ et travaillaient à temps complet ou à temps partiel $(n=9)$.

Tableau 1. Caractéristiques sociodémographiques des participants $(n=22)$

\begin{tabular}{lcc}
\hline \hline Caractéristiques & $\begin{array}{c}\text { Ados et jeunes } \\
\text { sociodémographiques }\end{array}$ & Parents $(\mathrm{n}=12)$ \\
\hline Sexe & 6 & 1 \\
- Masculin & 4 & 11 \\
- Féminin & $19,6(18-21)$ & $50,8(41-63)$ \\
Âge moyen (min.-max.) & & \\
Plus haut niveau de scolarité atteint & 2 & 0 \\
- Primaire & 3 & 0 \\
- Secondaire 1-2-3 & 4 & 0 \\
- Secondaire 4-5 & 0 & 4 \\
- Secondaire général complété & 1 & 0 \\
- Formation de préparation au travail & 0 & 3 \\
- Professionnel et collégial & 0 & \\
- Universitaire & & 0 \\
Occupation principale ( $\mathrm{n}=11$ parents) & 6 & 0 \\
- Études à temps complet & 2 & 4 \\
- Études à temps partiel & 0 & 5 \\
- Travail à temps complet & 2 & 2 \\
- Travail à temps partiel & 0 & \\
- À la maison/en recherche d'emploi & & \\
Sources de revenu principales & 3 & \\
- Travail & 2 & \\
- Sécurité du revenu & & \\
- Aide financière familiale & & \\
- Tâches rémunérées à la maison & & \\
\hline \hline
\end{tabular}




\section{Réalisation des habitudes de vie}

Les chercheurs ont observé des changements significatifs ( $p=0,001$ à 0,027$)$ chez les adolescents et jeunes adultes pour sept des neuf habitudes de vie : les déplacements, la nutrition, les soins personnels et de santé, l'habitation, les responsabilités, le travail et les activités physiques et de loisirs (tableau 2). Ceci signifie que la réalisation de ces activités a changé entre le début et la fin de l'intervention. L'analyse des résultats révèle que la perception des parents s'était aussi modifiée de manière positive $(p=0,000$ à 0,018$)$, concernant la réalisation de huit habitudes de vie sur neuf : la communication, les déplacements, la nutrition, les soins personnels et de santé, les responsabilités, les relations interpersonnelles, le travail et les activités physiques et de loisirs (tableau 2).

\section{Sentiment d'efficacité personnelle et estime de soi}

À l'égard du sentiment d'efficacité personnelle, l'échelle est constituée de deux facteurs, le sentiment d'efficacité personnelle général et social. Globalement, cette mesure indique une amélioration significative du sentiment d'efficacité chez les participants entre le début et la fin de l'intervention $(p=0,018)$ (tableau 2). Il en va de même pour la sous-échelle mesurant le sentiment d'efficacité général $(p=0,029)$. L'intervention ne semble pas avoir entraîné de changement significatif sur le sentiment d'efficacité social $(p=0,441)$ et sur le niveau d'estime de soi des participants $(p=0,236)$.

\section{Discussion}

Le but du présent article était de présenter les résultats quantitatifs recueillis lors d'une étude exploratoire visant à documenter et à évaluer, du point de vue des adolescents et jeunes adultes et de leur famille, les retombées d'interventions de groupe visant le soutien à la transition vers la vie adulte. L'équipe de recherche avait formulé des hypothèses à l'effet que les personnes ayant participé aux activités: 1) seraient davantage aptes à réaliser leurs habitudes de vie; 2) auraient un meilleur sentiment d'efficacité personnelle et un niveau plus élevé d'estime de soi à la fin (T2) comparativement au moment de l'entrée dans l'intervention (T0). 
Tableau 2. Niveau moyen de réalisation des habitudes de vie, du sentiment d'efficacité personnelle et de l'estime de soi et comparaison entre les valeurs obtenues au début et à la fin de l'intervention, jeunes $(n=10)$ et parents $(n=12)$

\begin{tabular}{|c|c|c|c|c|c|c|c|c|c|c|}
\hline \multirow[t]{2}{*}{ Habitude de vie } & \multicolumn{3}{|c|}{$\begin{array}{c}\text { Scores } \\
\text { moyens_jeunes }^{*}(n=10)\end{array}$} & \multirow{2}{*}{$\begin{array}{c}\text { Diff } \\
\text { moyenne } \\
\text { T0-T2 }\end{array}$} & \multirow{2}{*}{$\begin{array}{c}\text { Wilcoxon } \\
\text { T0-T2 } \\
p \text { exact } \\
\text { unilatéral }\end{array}$} & \multicolumn{3}{|c|}{$\begin{array}{l}\text { Scores moyens_parents* } \\
\qquad(n=12)\end{array}$} & \multirow{2}{*}{$\begin{array}{c}\text { Diff } \\
\text { moyenne } \\
\text { T0-T2 }\end{array}$} & \multirow{2}{*}{$\begin{array}{c}\text { Wilcoxon } \\
\text { T0-T2 } \\
p \text { exact } \\
\text { unilatéral }\end{array}$} \\
\hline & T0 & $\mathrm{T} 1$ & $\mathrm{~T} 2$ & & & T0 & $\mathrm{T} 1$ & $\mathrm{~T} 2$ & & \\
\hline Communication (9 items) & 9,50 & 9,78 & 9,79 & 0,29 & 0,057 & 8,85 & 9,44 & 9,58 & 0,73 & 0,000 \\
\hline Déplacements (6 items) & 7,71 & 8,87 & 9,03 & 1,28 & 0,004 & 6,94 & 9,03 & 8,95 & 2,01 & 0,000 \\
\hline Nutrition (4 items) & 8,39 & 9,22 & 9,50 & 1,14 & 0,014 & 8,27 & 9,37 & 9,51 & 1,24 & 0,000 \\
\hline $\begin{array}{l}\text { Soins personnels et de } \\
\text { santé ( } 6 \text { items) }\end{array}$ & 8,33 & 9,24 & 9,25 & 0,93 & 0,001 & 7,63 & 9,06 & 8,97 & 1,35 & 0,001 \\
\hline Habitation (1 items) & 7,30 & 8,55 & 9,20 & 1,90 & 0,004 & 7,29 & 8,86 & 8,29 & 1,00 & 0,131 \\
\hline Responsabilités (4 items) & 8,80 & 9,12 & 9,42 & 0,61 & 0,016 & 8,00 & 9,29 & 8,98 & 0,98 & 0,018 \\
\hline $\begin{array}{l}\text { Relations interpersonnelles } \\
\text { (3 items) }\end{array}$ & 9,48 & 9,77 & 9,72 & 0,23 & 0,125 & 8,58 & 9,21 & 9,32 & 0,74 & 0,001 \\
\hline Travail (3 items) & 7,72 & 9,12 & 9,07 & 1,35 & 0,027 & 7,42 & 9,13 & 8,94 & 1,24 & 0,012 \\
\hline Loisirs (8 items) & 8,10 & 9,03 & 9,52 & 1,42 & 0,001 & 8,21 & 8,98 & 9,18 & 0,97 & 0,001 \\
\hline $\begin{array}{l}\text { Sentiment d'efficacité } \\
\text { personnelle_score total }\end{array}$ & 3,38 & 3,59 & 3,60 & 0,22 & 0,018 & & & & & \\
\hline Échelle générale & 3,34 & 3,64 & 3,66 & 0,32 & 0,029 & & & & & \\
\hline Échelle sociale & 3,46 & 3,51 & 3,48 & 0,03 & 0,441 & & & & & \\
\hline Estime de soi & 2,96 & 3,16 & 3,06 & 0,06 & 0,236 & & & & & \\
\hline
\end{tabular}

* Score maximal de 10 pour le niveau de réalisation des habitudes de de vie; score maximal de 5 pour le sentiment d'efficacité personnelle et score maximal de 4 pour l'estime de soi. 
Cette première évaluation illustre que les interventions proposées par les intervenants sont associées à des changements significatifs $(p=0,001$ à 0,027$)$ dans la réalisation d'habitudes de vie par les adolescents et jeunes adultes, surtout en ce qui concerne les déplacements, la nutrition, l'habitation, les responsabilités et les activités physiques et de loisirs. L'évaluation des parents appuie la plupart de ces résultats, à l'exception de l'habitation et de la communication où les résultats sont différents (habitation : changement significatifs chez les jeunes et non chez les parents; communication : changement significatifs chez les parents et non chez les jeunes). En ce qui concerne la communication, cette différence s'explique par des scores distants entre parents et jeunes à l'entrée dans l'étude (score plus faible chez les parents). Quant à lui, l'aspect habitation comportait trop peu d'items.

Pour les déplacements, l'item ayant le plus grand changement perçu entre T0 et T2 (moyenne des différences entre les scores; résultats non montrés) est le même pour les jeunes et les parents, soit « Utiliser des moyens de transport comme passager (auto, transport en commun, taxi) ». En ce qui concerne la nutrition, il en est de même avec l'item "Choisir les aliments pour vos repas, selon vos goûts et vos besoins particuliers (quantité, fraîcheur, type d'aliments, diète personnelle, faire l'épicerie) ». Quant aux responsabilités, l'item ayant enregistré le plus grand changement est "Planifier votre budget et respecter vos obligations financières (répartir vos dépenses et vos économies, paiement des comptes) ». Ce sont tous des aspects qui ont pu être directement influencés par les interventions de groupe par le biais d'activités telles que repas au restaurant, pique-nique, planification du budget en vue de la participation aux activités ou utilisation du transport en commun. En ce qui a trait aux résultats liés au travail ainsi qu'aux loisirs, les chercheurs considèrent qu'ils doivent être interprétés avec prudence en raison de données manquantes tant chez les parents que chez les participants.

Les interventions proposées s'accompagnent aussi de changements positifs significatifs sur le sentiment d'efficacité personnelle des participants, c'est-à-dire leur sentiment d'avoir les capacités nécessaires pour atteindre des résultats désirés. Les items ayant le plus grand changement perçu entre T0 et T2 (moyenne des différences entre les scores; résultats non montrés) en lien avec la sous-échelle d'efficacité personnelle générale sont: "Un de mes problèmes est que je ne peux pas me mettre au travail lorsqu'il faudrait »; "Quand j'établis des objectifs qui sont importants pour moi, il est rare que je les atteigne "; " J'évite de faire face aux difficultés »; "Quand je décide de faire quelque chose, je m'y consacre immédiatement »; "Quand j'essaie d'apprendre quelque chose de nouveau, j'abandonne très vite si je n'y arrive pas tout de suite »; "Quand des problèmes inattendus surviennent, j'arrive bien à y faire face et finalement », « J'abandonne facilement ».

D'autres études ont évalué des interventions visant à faciliter la transition vers la vie adulte d'adolescents ayant des incapacités (Doren et al., 2013; Keenan et al., 2014). De ces interventions ont émergé des caractéristiques favorables correspondant à l'approche et aux principes mis de l'avant dans le cadre des services de "Soutien à la transition vers la vie adulte ». Parmi celles-ci, il y a l'adoption d'une approche centrée sur les personnes, qui permette de faire des apprentissages in vivo, dans la communauté, et basée sur l'expérience. Dans le cadre de la présente étude, cela signifie l'implication des individus afin qu'ils puissent communiquer, planifier et gérer les démarches à faire, prendre des décisions, faire des choix dans l'organisation d'activités menées dans la communauté. Une approche centrée sur les 
personnes favorise la participation à la prise de décisions et l'expression des idées et des opinions alors que les stratégies d'apprentissage en communauté et basées sur l'expérience ont l'avantage d'impliquer une expérience réelle, sont associées au succès, et dont les acquis sont directement applicables à d'autres aspects de la vie. Ces stratégies favorisent la participation active et permettent de vivre de nouvelles expériences, de vivre de petits succès et de prendre des risques calculés, ce qui a des effets bénéfiques sur l'autodétermination et le sentiment d'efficacité personnelle (Doren et al., 2013; Keenan et al., 2014). La présence de différences déjà notables au $\mathrm{T} 1$ tant chez les jeunes et que chez les parents pour la réalisation de certaines habitudes de vie (déplacements, nutrition, soins personnels et de santé) amènent les auteurs à se questionner sur la durée de l'intervention. Une intervention d'une durée de 6 mois, plutôt que 12, serait-elle suffisante pour produire des résultats positifs significatifs chez les jeunes en ce qui concerne la réalisation de ces habitudes de vie?

L'intervention proposée par les intervenants n'est toutefois pas parvenue à influencer de manière positive et significative leur niveau d'estime de soi. Ces résultats peuvent peut-être s'expliquer par le fait que les intervenants n'avait pas ciblé cet aspect spécifiquement par l'intervention, mais également par le fait que l'estime de soi est en partie le résultat d'expériences antérieures de succès ou d'échec (Sherer et al., 1982). Or, les personnes ayant des incapacités manquent souvent d'occasions de participer ou d'exercer un contrôle dans diverses activités de la vie quotidienne (East et Orchard, 2014). II est possible d'affirmer qu'il leur faudra sans doute quelques expériences de succès supplémentaires pour influencer de manière positive leur estime d'eux-mêmes. De plus, l'utilisation d'un questionnaire mesurant mieux la réalité des jeunes et mesurant plusieurs aspects associés à l'estime de soi (p. ex. : sur les plans de l'apparence physique, social, familial), plutôt qu'un seul, aurait peut-être été plus appropriée.

Les résultats révèlent que les interventions proposées n'ont pu influencer de manière positive et significative le sentiment d'efficacité personnelle en lien avec les situations sociales. En fait, certaines personnes ont eu une amélioration ( $\mathrm{J} 1, \mathrm{~J} 5, \mathrm{~J} 7)$ alors que d'autres ont eu une baisse $(\mathrm{J} 4, \mathrm{~J} 6, \mathrm{~J} 10)$. Les items les plus souvent influencés par l'intervention, de manière positive ou négative, sont : «Si j'aperçois quelqu'un que j'aimerais rencontrer, je vais vers cette personne plutôt que d'attendre qu'elle vienne vers moi » et l'item «Je n'arrive pas à me comporter comme je le voudrais lors de réunions sociales (fêtes, repas, apéritifs, etc.) ». Le fait de participer plus activement à des activités sociales a peut-être amélioré pour certains leur ouverture vers les autres et leur sentiment de se comporter selon les convenances sociales. À l'inverse, pour d'autres, ces occasions ont peut-être entraîné une prise de conscience de leurs différences et de leur manque de connaissance des convenances sociales, entraînant un repli sur soi et un sentiment de ne pouvoir se comporter convenablement lors d'événements sociaux.

II est certain que la répétition de ces expériences pourra à long terme entraîner des résultats positifs au plan de l'efficacité personnelle en situation sociale. Les résultats de cette étude exploratoire montrent qu'il est possible de favoriser, à l'aide d'interventions de groupe, l'acquisition d'habiletés essentielles à la transition vers la vie adulte pour les personnes ayant des incapacités. Toutefois, cette étude et d'autres du même type peuvent susciter des questionnements plus larges sur la signification de la vie adulte pour ces individus. En raison de la présence d'incapacités, à laquelle s'ajoute parfois une espérance de vie limitée, les pratiques 
et les normes associées à la vie adulte doivent-elles être mises de l'avant pour ces jeunes ? Sur la base de quels critères doit-on établir leur satisfaction sociale ? Est-ce qu'ils doivent être encouragés ou dissuadés de quitter l'univers familial? (Gibson et al., 2014). Ces questionnements sont légitimes et requièrent une réflexion impliquant tous les acteurs.

D'un autre côté, en regard du discours que les adolescents et jeunes adultes ayant des incapacités tiennent sur leur propre développement, il est tout aussi pertinent de remettre en question les conceptions restrictives à leur endroit et de mettre à leur disposition des lieux d'apprentissage qui prennent en compte leurs caractéristiques. Ceux-ci leur permettront de répondre à leurs désirs et aspirations, d'acquérir des habiletés cognitives et comportementales et de réaliser des étapes essentielles dans le cheminement vers la vie adulte, des aspects cruciaux d'une vie satisfaisante et d'une participation sociale réussie. De plus, ces aspects contribuent positivement à la santé et au bien-être (Sattoe et al., 2014).

Cela nécessite de la part des parents et des professionnels de changer les rôles, c'est-à-dire de changer les manières de penser et d'agir à leur endroit, d'une vision axée sur le contrôle vers une qui serait axée sur l'accompagnement dans le développement des habiletés de gestion et de participation sociale. La redirection ne doit cependant pas faire fi de l'implication déjà importante des familles et de l'effet négatif des incapacités des adolescents et jeunes adultes sur leur réalité (Stewart et al., 2013). La vie avec un handicap est déjà marqué par la présence de préoccupations, de tensions, de stress, d'épuisement et d'isolement social vécus par les familles (Bristol et Schopler, 1983; Cimon et al., 2002; Dionne et Rousseau, 2006; Duvdevany, 2001). Les bénéfices potentiels pour les enfants ne doivent pas se traduire en fardeau supplémentaire pour les familles, surtout en terme de temps et de déplacements (Crompton et al., 2007 dans Boucher et McGinn, 2012). Ici les chercheurs sont d'avis, après discussions avec des intervenantes du programme, que le travail social est directement interpellé afin de jouer un rôle clé dans ce processus d'accompagnement des parents en contexte de transition vécue par les adolescent et jeunes adultes ayant des incapacités. Dans la mesure où les attentes et les projets de vie diffèrent entre les deux, le recours à une approche systémique est tout à fait approprié afin d'impliquer les parents et leur adolescent ayant des incapacités (Sattoe et al., 2014).

\section{Limites}

Cette étude repose sur un nombre relativement restreint de jeunes participants $(n=10)$. De plus, l'intervention qu'elle a mesurée était peu structurée et ne comportait pas d'objectifs individuels spécifiques. Étant donné ces deux éléments, il est difficile de tirer des conclusions sur l'apport réel de cette intervention au développement de compétences utiles au passage à la vie adulte chez des jeunes en situation d'incapacité physique. Toutefois, des résultats positifs significatifs semblent en émerger.

\section{Conclusion}

Le but de cette étude était d'abord et avant tout de documenter et d'évaluer de manière plus systématique les retombées des interventions de groupe offertes dans le cadre des services de «Soutien à la transition vers la vie adulte » du CIUSSS de la Capitale-Nationale - IRDPQ. Les 
informations obtenues devaient constituer le point de départ d'une réflexion de la part des intervenants et des gestionnaires à l'endroit des modalités d'intervention. L'étude a révélé dans son ensemble des résultats positifs significatifs en ce qui concerne la réalisation de certaines habitudes de vie par les adolescents et jeunes adultes, notamment en ce qui a trait à leurs capacités à utiliser les transports et à planifier un budget, résultats en très grande partie appuyés par les perceptions des parents. Les résultats démontrent aussi une amélioration du sentiment d'efficacité personnelle à atteindre des buts fixés. L'approche et les principes mis de l'avant dans ce programme, c'est-à-dire une approche centrée sur les adolescents et jeunes adultes, permettant de faire des apprentissages in vivo, dans la communauté, et basée sur l'expérience, sont certainement en partie responsables de ces résultats positifs puisqu'ils ont été identifiés par des auteurs dans les écrits scientifiques comme des conditions d'intervention favorables au développement des jeunes ayant des incapacités. Cette étude a aussi permis de mettre en lumière des changements nécessaires au programme, notamment définir des objectifs spécifiques pour chacun des jeunes, planifier davantage les activités en fonction des objectifs du groupe, proposer un calendrier préétabli des activités, graduer la complexité des sorties, développer des ateliers d'apprentissage préalables aux activités et développer des interventions de groupe pour les parents. Étant donné les limites de la présente étude ainsi que les changements apportés au programme, une étude subséquente, comprenant davantage de participants ainsi que des entrevues individuelles, permettrait de mieux identifier les retombées du programme sur le développement d'habiletés pertinentes au passage à la vie adulte pour les adolescents et jeunes adultes ayant des incapacités physiques.

BOUCHER, Normand

SCHMOUTH, Marie-Ève

LIZOTTE, Andrée-Anne

TÉTREAULT, Sylvie

CÔTÉ, J.

COUTURE, M.

\section{RÉFÉRENCES}

Betz, C.L., et G. Redcay (2002). «Lessons learned from providing transition services to adolescents with special health care needs ", Issues in Comprehensive Pediatric Nursing, vol. 25, no 2, p. 129-149. doi: 10.1080/01460860290042549.

Blascovich, J., et J. Tomaka (1991). " Measures of self-esteem », dans J.P. Robinson et P.R. Shaver (dir.), Measures of personality and social psychological attitudes, San Diego (CA), Academic Press, p. 115-160. 
Blum, R.W. (1995). «Transition to adult health care: setting the stage », Journal of Adolescent Health, vol. 17, no 1, p. 3-5. doi: 10.1016/1054-139X(95)00073-2.

Boucher, N., D. Fiset, Y. Lachapelle, P. Fougeyrollas, avec la collaboration de L. Noreau et J. Leblond (2013). Projet d'expérimentation de la MHAVIE en sites-pilotes des centres de réadaptation en déficience physique et intellectuelle, trouble envahissant du développement et des centres de santé et de services sociaux, volet soutien à domicile, Québec, Institut de réadaptation en déficience physique de Québec, Centre interdisciplinaire de recherche en réadaptation et intégration sociale.

Boucher, N., et C.A. McGinn (2012). Note brève. Les interventions thérapeutiques de groupe visant le développement des compétences sociales et des capacités motrices des enfants et des adolescents avec une déficience physique : identification des conditions gagnantes d'organisation, Québec, Unité d'évaluation des technologies et des modes d'intervention en santé et en services sociaux (UETMISSS), Institut de réadaptation en déficience physique de Québec, 18 pages.

Bristol, M.M., et E. Schopler (1983). "Stress and coping in families with autistic adolescents », dans E. Schopler et G.B. Mesibov (dir.), Autism in adolescents and adults. New York (NY), Plenum Publishing Corp., p. 251-278.

Cimon, L., P. Beaupré et S. Tétreault (2002). « Expérimentation d'un programme de transition à la vie adulte pour des jeunes ayant une déficience physique : Impact sur l'intervention auprès des familles », dans S. Tétreault, P. Beaupré, J. C. Kalubi et B. Michallet (dir.), Famille et situation de handicap. Comprendre pour mieux intervenir. Sherbrooke (QC), CRP Éditeur, p. 75-89.

Dionne, C., et N. Rousseau (2006). Évaluation qualitative de la situation des familles où vit une personne handicapée, Drummondville (QC), Office des personnes handicapées du Québec (OPHQ).

Doren, B., M.-C. Yan, et W.-M. Tu (2013). « Key program features to enhance the school-tocareer transition for youth with disabilities », Prevention Researcher, vol. 20, no 2, p. 11-13.

Dorval, G. (1996). Impact de deux programmes aquatiques chez une clientèle adolescente ayant une déficience motrice cérébral, Maîtrise en médecine expérimentale, Université Laval.

Duvdevany, I. (2001). « Parents of adult children with developmental disabilities: Stress, social support and their views of normalization in relation to their attitudes toward their children's employment», International Journal of Rehabilitation Research, vol. 24, no 3, p. 227-232.

East, L.J., et T.R. Orchard (2014). " "Why can't I?": an exploration of sexuality and identity among Canadian youth living with physical disabilities », Journal of Youth Studies, vol. 17, no 5, p. 559-576. 
Fleming, J.S., et B.E. Courtney (1984). « The dimensionality of self-esteem: II. Hierarchical facet model for revised measurement scales », Journal of Personality and Social Psychology, vol. 46 , no 2 , p. 404-421.

Fougeyrollas, P., L. Noreau et al. (2011). La Mesure des habitudes de vie (MHAVIE 4.0). Version pour adolescents, adultes et aînés, Québec, Réseau international sur le Processus de production du handicap (RIPPH).

Fougeyrollas, P., L. Noreau et al. (2012). Guide d'utilisation de la mesure des habitudes de vie (MHAVIE 4.0). Version pour adolescents, adultes et aînés, Québec, Réseau international sur le Processus de production du handicap (RIPPH).

Genest, M.-E. (s. d.). L'échelle des perceptions d'efficacité personnelle, Centre d'études et de recherche en intervention familiale, Université du québec en Outaouais. Accédé le 6 juillet 2015, <http://cerif.uqo.ca/l\%E2\%80\%99\%C3\%A9chelle-des-perceptionsd\%E2\%80\%99efficacit\%C3\%A9-personnelle>.

Gibson, B.E., B. Mistry, B. Smith, K.K. Yoshida, D. Abbott, S. Lindsay et Y. Hamdani (2014). «Becoming men: Gender, disability, and transitioning to adulthood », Health (London), vol. 18, no 1, p. 95-114. doi: 10.1177/1363459313476967.

Keenan, S., G. King, C.J. Curran et A. McPherson (2014). « Effectiveness of experiential life skills coaching for youth with a disability », Physical and Occupational Therapy in Pediatrics, vol. 34, no 2, p. 119-131. doi: 10.3109/01942638.2013.842200.

Magill-Evans, J. (1993). Measures of self-esteem for children and adolescents, communication présentée dans le cadre du Congress of the American and Canadian Occupational Therapy Association, Boston (MA).

Reiter, S., et T. Goldman (1999). «A programme for the enhancement of autonomy in young adults with physical disabilies: the development of a realistic self concept, individual perception of quality of life and the development of independent living skills ", International Journal of Rehabilitation Research, vol. 22, no 1, p. 71-74.

Rosenberg, M. (1965). Society and the adolescent self-image, Princeton (NJ), Princeton University Press.

Sattoe, J.N., S.R. Hilberink, A. van Staa et R. Bal (2014). « Lagging behind or not? Four distinctive social participation patterns among young adults with chronic conditions », Journal of Adolescent Health, vol. 54, no 4, p. 397-403. doi: 10.1016/j.jadohealth.2013.09.017.

Sherer, M., et C.H. Adams (1983). "Construct Validation of the Self-efficacy Scale ", Psychological Reports, vol. 53, no 3, p. 899-902. 
Sherer, M., J.E. Maddux, B. Mercandante, S. Prentice-Dunn, B. Jacobs et R.W. Rogers (1982).

"The Self-efficacy Scale: Construction and validation », Psychological Reports, vol. 51, no 2, p. 663-671.

Skär, L. (2003). «Peer and adult relationships of adolescents with disabilities », Journal of Adolescence, vol. 26, no 6, p. 635-649.

Stevenson, C.J., P.O. Pharoah et R. Stevenson (1997). "Cerebral palsy-the transition from youth to adulthood », Developmental Medicine and Child Neurology, vol. 39, no 5, p. 336342.

Stewart, D., J.W. Gorter et M. Freeman (2013). " Transitions to adulthood for youth with disabilities: Emerging themes for practice and research ", Prevention Researcher, vol. 20, no 2, p. 3-6. 